\title{
Motor competence in Czech children aged 11-15: What is the incidence of a risk of developmental coordination disorder?
}

\author{
Jakub Kokštejn ${ }^{1, *}$, Rudolf Psotta ${ }^{2}$, and Martin Musálek ${ }^{1}$ \\ ${ }^{1}$ Faculty of Physical Education and Sport, Charles University, Prague, Czech Republic; ${ }^{2}$ Faculty of Physical Culture, Palacký \\ University Olomouc, Olomouc, Czech Republic
}

Copyright: (C) 2015 J. Kokštejn et al. This is an open access article licensed under the Creative Commons Attribution License (http://creativecommons.org/licenses/by/4.0/).

\begin{abstract}
Background: Current findings suggest that the prevalence of developmental coordination disorder (DCD) ranges widely between countries. A major reason for this wide range of prevalence is how cases of DCD are identified. Gender differences in level of motor competence in children with movement difficulties may play a key role in the choice of type of intervention. Objective: The aim of the study was to reveal the prevalence of significant movement difficulties with high probability of presence of DCD in Czech children aged 11 to 15 . At the same time we wanted to assess possible gender differences in different types of the movement difficulties. Methods: A total sample of 507 children (age 11-15 years, 262 boys, 245 girls) from all Czech regions was included. The MABC-2 test was used for the identification of movement difficulties with different severity. Children whose total test score (TTS) was $\leq 15^{\text {th }}$ percentile were considered at risk for having DCD (children with rDCD). Children whose TTS was $\leq 5^{\text {th }}$ percentile were considered as having significant movement difficulties with high probability of presence of DCD. An analysis of gender differences of children with rDCD in MABC-2 motor components and tests were carried out. Results: From the entire sample, 33 participants ( 22 boys, 11 girls) were identified as at risk of having DCD (rDCD). $1.4 \%$ of the total sample met the criterion for significant movement difficulties with high probability of presence of DCD. 5.1\% of the total sample met the criterion for identification of the risk for having movement difficulties. Almost twice as high predisposition for the occurrence of movement difficulties was revealed in boys as compared to girls in a population of children with $\mathrm{rDCD}(O R=1.95,95 \% \mathrm{CI}: 1.16-2.74)$. Girls with $\mathrm{rDCD}$ performed better in manual dexterity with a medium effect of the gender (Cohen's $d=0.58$ ), whereas boys with $\mathrm{rDCD}$ achieved better results in aiming and catching also with a medium effect (Cohen's $d=0.50$ ). Nevertheless, statistically significant differences in these components have not been confirmed. Conclusion: We found a $1.4 \%$ incidence of significant movement difficulties among Czech children. A significantly higher incidence of developmentally conditioned movement difficulties were found in boys. Gender differences in the level of motor skills of children with rDCD reflect a common trend in the general child population.
\end{abstract}

Keywords: movement difficulties, prevalence of DCD, fine motor coordination, balance, gender differences

\section{Introduction}

The importance of motor competence as a basic precondition for physical, social and cognitive development in children has been documented in detail (Haywood \& Getchell, 2009; Pine, Lufkin, \& Messer, 2004). Acquisition of motor skills are an essential part of total development in children; and thus they become

\footnotetext{
* Address for correspondence: Jakub Kokštejn, Department of Sport Games, Faculty of Physical Education and Sport, Charles University, José Martího 31, 16252 Praha 6 - Veleslavín, Czech Republic. E-mail: kokstejn@ftvs.cuni.cz
}

more competent and skilful (Goodway \& Branta, 2003; Henniger, 2008). The insufficient level of motor competence leads to lower achievement in school, social isolation and various emotional and behavioural problems at a later age (Henderson, Sugden, \& Barnett, 2007). According to occupational therapists and physiotherapists, there are today more school-age children who have problems with writing, suffer from developmental delays in fine and gross motor skills, have problems with balance and are generally clumsy (Missiuna, 2001).

After specialized examination, such children are sometimes diagnosed with developmental coordination 
disorder (DCD). DCD is now a globally used term in Diagnostic and Statistical Manual of Mental Disorders, Fifth Edition (DSM-V; APA, 2013) for individuals whose acquisition and execution of coordinated motor skills are substantially below that which is expected for the patient's age and opportunities for learning. Clumsiness and slow inaccurate performance of motor skills are present, and interfere with activities of daily life and impact academic, occupational, recreational or social activities (APA, 2013). Delayed or immature motor development may interfere, alone or in combination, with fine motor skills, gross motor skills and balance or postural stability (APA, 2000; Barnhart, Davenport, Epps, \& Nordquist, 2003; Chambers \& Sugden, 2006). Typical DCD manifestations include functional problems in the body scheme, in perception of space and body position in space (Wann, Williams, \& Rushton, 1998), sensory integration (Elbasan, Kayihan, \& Duzgun, 2012), problems in bimanual and visuo-manual coordination (Volman \& Geuze, 1998), and visual memory (Dwyer \& McKenzie, 1994).

The social impact of DCD is mostly related to its prevalence which, according to the current estimates, ranged between 2\% and 4\% (Barnhart et al., 2003; Gaines, Missiuna, Egan, \& McLean, 2008; Lingam, Hunt, Golding, Jongmans, \& Emond, 2009; Tsiotra et al., 2006;). On the other hand, Lingam et al. (2009) and Blank, Smits-Engelman, Polatajko, and Wilson (2012) have been critical of this, stating that the wide prevalence range of DCD is due to the selection of inconsistent criteria for diagnosing DCD. The fact that the criteria for DCD diagnosis are not strictly observed may be another problem. Lingam et al. (2009) further have stated that high DCD prevalence in different studies reflected the number of children that failed a motor test than the real number of children with serious motor impairment which negatively affects success in performing activities of daily living.

In the Czech Republic, the problem of developmentally conditioned movement difficulties and the possible occurrence of DCD among Czech children has been studied by Psotta and Hendl (2012), Psotta, Hendl, Frömel, and Lehnert (2012) and Psotta, Kokštejn, and Vodička (2009). Results of the first two studies suggested prevalence of significant movement difficulties in Czech school-age children (0.6-1.9\%), and prevalence of the risk of having movement difficulties (1.6-4.7\%). Psotta and Hendl (2012) also claimed that to date there was no universally used diagnostic method assessing the level of motor coordination in children in the Czech Republic.

Macnab, Miller, and Polatajko (2001) emphasized a frequent overlap between DCD and other disorders. This comorbidity is one of the main causes of problems with the classification of the children with DCD. Up to $50 \%$ of the children with DCD are concurrently diagnosed with learning disorders such as dyslexia, dysgraphia, dysorthography and dyscalculia, as well as ADHD and speech impairments (Kadesjo, 1999; Martin, Piek, \& Hay, 2006; Watemberg, Waiserberg, Zuk, \& Lerman-Sagie, 2007).

According to the older studies, DCD prevalence is higher in boys in comparison to girls, with the ratio ranging between 3:1 and 7:1 (Cermak \& Larkin, 2002; Gillberg, 2003; Kadesjö \& Gillberg, 1998; Kourtessis et al., 2008; Missiuna et al., 2008). However, epidemiological research $(N=7000)$ in English school-aged population revealed lower gender ratio 1.9:1 (Lingam et al., 2009). General gender differences in motor skilfulness in children and youth have been well documented (Booth, Okely, Denney-Wilson, Hardy, Yang, \& Dobbins, 2006; Ehl, Roberton, \& Langendorfer, 2005; Thomas \& French, 1985). While pre-school children's gender differences are minimal in achievement of motor milestones (Malina, Bouchard, \& Bar-Or, 2004), at older school age and adolescence significant gender differences have been clearly proven, especially in the gross motor skills (Barnett, Van Beurden, Morgan, Brooks, \& Beard, 2010; Junaid \& Fellowes, 2006; Lorson \& Goodway, 2008; van Beurden, Zask, Barnett, \& Dietrich, 2002). A wide range of studies have observed significantly better results in boys in comparison with girls in motor skills that require control of larger objects, e.g., catching, kicking, and throwing at a target (Barnett et al., 2010; Butterfield, Angell, \& Mason, 2012; Junaid \& Fellowes, 2006; Malina et al., 2004).

As far as the manual abilities are concerned, considerable disagreement exists with respect to gender differences. Some studies have revealed significantly better results in fine motor activities in the school population of boys in comparison to girls (Dorfberger, AdiJapha, \& Karni, 2009; Gur et al., 2012). By contrast, Junaid and Fellowes (2006) and Poole et al. (2005) have observed significantly better results in fine motor activities in girls.

With respect to gender differences in balance skills, only a few studies detected significant differences between boys and girls (Nolan, Grigorenko, \& Thorstensson, 2005). Related to the problem of prevalence of DCD among the children presented above, the aim of the study was to investigate the prevalence of developmentally conditioned movement difficulties in Czech children aged 11 to 15 years old. At the same time we wanted to assess possible gender differences in the different types of movement difficulties. 


\section{Methods}

\section{Participants}

A total sample of 507 children aged 11-15 years (262 boys, 245 girls; $M=12.9$ years, $S D=1.2$ ) from all Czech regions were included in the study (Table 1). Children were randomly selected from twelve primary schools. The study was conducted in accordance with the Declaration of Helsinki. The testing of children was carried out after receiving informed written consent from the school's principal and the children's parents. Children already diagnosed with mental or general medical impairments (according to $\mathrm{C}$ and $\mathrm{D}$ criteria for DCD; APA, 2000) were not included in the measurement.

Table 1

Numerical and descriptive data of participants $(N=507)$

\begin{tabular}{lcc}
\hline Age (years) & Boys $(n)$ & Girls $(n)$ \\
\hline 11 & 66 & 70 \\
12 & 87 & 66 \\
13 & 60 & 62 \\
14 & 37 & 29 \\
15 & 12 & 18 \\
\hline
\end{tabular}

\section{Measurements}

The test of the Movement Assessment Battery for Children-2 (MABC-2 test; Henderson et al., 2007) was used for identification of movement difficulties. The MABC-2 test can be currently considered the most comprehensive diagnostic tool for the assessment of motor development and identification of movement difficulties with different severity (Henderson et al.). The MABC-2 test is designed for children in the different age bands $(A B)$ from 3 to 16 years old (AB1: 3-6 years; AB2: 7-10 years; AB3: 11-16 years). The AB3 covers the evaluation of three major motor coordination components with eight motor tasks overall:

1. Manual Dexterity (fine motor coordination): Turning pegs (MD1), Triangle with nuts and bolts (MD2), and Drawing trail (MD3).

2. Gross Motor Coordination: Catching with one hand (AC1) and Throwing at a wall target (AC2) for the assessment of aiming and catching.

3. Balance: Two-board balance (Bal1), Walking toe-toheel backwards (Bal2), and Zig-zag hopping (Bal3).

According to the Examiner's manual (Henderson et al., 2007) the raw scores achieved in each test item was converted to the age-normed standard score. In the MABC-2 test the overall level of fundamental motor skills is represented by the total test score (TTS), which is calculated as a sum of the standard scores of all eight test items, and its conversion to the standard score equivalent and percentile equivalent. MABC-2 test-retest reliability for TTS is very good with an intraclass correlation coefficient $I C C=.97, I C C=.88-$ .99 for individual test items and $I C C=.91-.97$ for the particular motor components in children 6-12 years old (Chow, Chan, Chan, \& Lau, 2002; Wuang, Su, \& $\mathrm{Su}, 2012)$. According to the same authors the internal consistency for the MABC-2 Test was $\alpha=.90$.

TTS at or below the $5^{\text {th }}$ percentile is the standard cut-off score for significant movement difficulties and serves as an indicator for high probability of presence of DCD in a child. TTS between the $6^{\text {th }}$ and $15^{\text {th }}$ percentile is considered the indicator of the risk of movement difficulties, and TTS at above the $15^{\text {th }}$ percentile is consistent with typical motor coordination development (Henderson et al., 2007). TTS below $16^{\text {th }}$ percentile is also usually used as the indicator of a risk of DCD (rDCD) (Cheng et al., 2014; Johnson \& Wade, 2009; Kanioglou, 2006; Schott, Alof, Hultsch, \& Meermann, 2007). In our study we adopted this diagnostic approach.

\section{Procedure}

The testing of the children was done by a team of trained research assistants with Master's degrees in physical education and sport, special pedagogy or adapted physical education. Children's motor skills were tested in schools during the morning classes. The fine motor skills testing, including measurement of body height and weight, were completed in a room selected for this purpose (presence of tables and chairs). The gross motor skills and balance testing were examined in a gym.

\section{Statistical methods}

The odds ratio statistical method $(\mathrm{CI}=95 \%)$ was used to express gender ratios in prevalence of children with movement difficulties according to performance in MABC-2. The Kolmogorov-Smirnov test was used for testing normality of data distribution $(p \leq .05)$. To test the practical and statistical significance of gender differences in TTS, each motor component and each test item were analysed. The effect size was tested using the Cohen's coefficient of the effect size $(d)$, using pooled $S D$ (Cohen, 1988). The value $d<0.50$ was interpreted as a small effect, $d=0.50-0.80$ as a moderate effect, and $d>0.80$ as a large effect (Cohen, 1988). The statistical significance of the difference was tested by the two sample $t$-test $(p \leq .05)$ including the $F$-test $(p \leq .05)$. The analysis was performed using NCSS Statistical Software (Version 2007; NCSS, Kaysville, UT, USA). 


\section{Results}

From the entire sample, 33 participants $(6.5 \% ; 22$ boys and 11 girls) were identified with a risk of DCD (rDCD; $\leq 15^{\text {th }}$ percentile; Table 2 ). From those six boys and one girl, presenting $1.4 \%$ of the total sample met the criterion for significant movement difficulty (TTS $\leq 5^{\text {th }}$ percentile). $5.1 \%$ of the total sample (16 boys and 10 girls) met the criterion for a risk of having movement difficulty (TTS $6-15^{\text {th }}$ percentile). Boys had significantly greater probability of movement difficulties than girls in a ratio of almost 2:1 $(O R=1.95,95 \%$ CI: 1.16-2.74).

The effect sizes were found in the motor components of motor dexterity and aiming and catching between boys and girls with rDCD (Table 3). Girls with rDCD performed better in manual dexterity tasks with a medium effect of the gender (Cohen's $d=0.58$; $p=.12$ ), while boys with rDCD were better in aiming and catching tasks also with a medium effect (Cohen's $d=0.50 ; p=.08)$. However, these findings were not confirmed by statistically significant differences between boys and girls with rDCD. According to our meaning, the statistically insignificant differences in manual dexterity and aiming and catching tasks could probably be caused by a different number of boys and girls in rDCD group. No significant gender differences were found in the balance component of MABC-2.

Practical and statistically significant gender differences were found in some tasks on MABC-2 (Table 4).
In the component of manual dexterity girls with rDCD performed significantly better on MD3 task (Cohen's $d=1.15 ; p \leq .05$ ). Boys with rDCD were significantly better in both aiming and catching tasks (AC1 Cohen's $d=0.98 ; p \leq .05$; AC2 - Cohen's $d=0.59$ ). Boys with rDCD were also significantly better in balance component of MABC-2 in Bal2 task (Cohen's $d=1.22 ; p \leq .05)$. No significant differences were found in other tasks on MABC-2.

\section{Discussion}

The aim of the study was to investigate the incidence of a risk of developmental coordination disorder in Czech children ages 11 to 15 years old. At the same time, we set out to detect gender differences in individual motor skills components in children with rDCD. In our study, a total of 33 children (6.5\%) scored under or equal the $15^{\text {th }}$ percentile in the MABC-2 test and were labelled as rDCD group. Of the $507 \mathrm{Czech}$ children included in the study, definite significant movement difficulty with high probability of presence of DCD was diagnosed in $1.4 \%$ of the sample. Prevalence of significant movement difficulty observed in our study is similar to the $1.8 \%$ prevalence detected in the study by Lingam et al. (2009) and the $1.6 \%$ detected in the study by Kourtessis et al. (2008). Although expected DCD prevalence is $2-4 \%$ in the entire school children population (APA, 2013), data from other countries showed a much wider

Table 2

Incidence of movement difficulties according to TTS in MABC-2 in relation to gender

\begin{tabular}{lcccc}
\hline & $\begin{array}{c}\text { Risk of DCD (rDCD) } \\
\text { TTS } \leq 15^{\text {th }} \text { percentile }\end{array}$ & $\begin{array}{c}\text { Significant movement } \\
\text { difficulty } \\
\text { TTS } \leq 5^{\text {th }} \text { percentile }\end{array}$ & $\begin{array}{c}\text { At risk of movement } \\
\text { difficulty } \\
\text { TTS } 6-15^{\text {th }} \text { percentile }\end{array}$ & $\begin{array}{c}\text { Without movement } \\
\text { difficulty }\end{array}$ \\
\hline Total & $33(6.5 \%)$ & $7(1.4 \%)$ & $26(5.1 \%)$ & $15^{\text {th }}$ percentile \\
Boys & $22(8.4 \%)$ & $6(1.2 \%)$ & $16(3.1 \%)$ & 474 \\
Girls & $11(4.5 \%)$ & $1(0.2 \%)$ & $10(2 \%)$ & 240 \\
\hline
\end{tabular}

Note. $\quad \mathrm{rDCD}=$ risk of developmental coordination disorder $(O R=1.95 ; 95 \% \mathrm{CI}=1.16-2.74)$.

Table 3

Gender differences in standards score in each component of $M A B C-2$ in children with $r D C D$

\begin{tabular}{lcccc}
\hline Component of MABC-2 & Boys $(M \pm S D)$ & Girls $(M \pm S D)$ & $d$ & $p$ \\
\hline Manual dexterity & $7.6 \pm 2.3$ & $8.5 \pm 0.8$ & $\mathbf{0 . 5 8}^{*}$ & .12 \\
Aiming and catching & $7.5 \pm 2.2$ & $6.3 \pm 2.6$ & $\mathbf{0 . 5 0}^{*}$ & .08 \\
Balance & $6.1 \pm 1.9$ & $6.3 \pm 2.0$ & 0.10 & .40 \\
\hline
\end{tabular}

Note. $\quad d=$ Cohen's coefficient of effect size. * medium practical significant difference. 
Table 4

Gender differences in standard score in each test of $M A B C-2$ in children with $r D C D$

\begin{tabular}{lcclc}
\hline Tests of MABC-2 & Boys $(M \pm S D)$ & Girls $(M \pm S D)$ & $d$ & $p$ \\
\hline Manual dexterity & & & & \\
MD1 & $7.0 \pm 2.4$ & $6.7 \pm 1.8$ & 0.14 & .37 \\
MD2 & $6.5 \pm 3.2$ & $6.5 \pm 1.4$ & 0.00 & .48 \\
MD3 & $9.8 \pm 3.2$ & $12.5 \pm 1.5$ & $\mathbf{1 . 1 5}^{* *}$ & $\mathbf{. 0 0 6}$ \\
Aiming and catching & & & & \\
AC1 & $8.8 \pm 3.0$ & $6.4 \pm 1.9$ & $\mathbf{0 . 9 8}^{* *}$ & $\mathbf{. 0 1 0}$ \\
AC2 & $8.4 \pm 2.0$ & $7.1 \pm 2.4$ & $\mathbf{0 . 5 9 *}$ & .052 \\
Balance & & & & .13 \\
Bal1 & $6.9 \pm 2.9$ & $8.2 \pm 3.5$ & 0.41 & .006 \\
Bal2 & $6.4 \pm 3.5$ & $3.5 \pm 1.3$ & $\mathbf{1 . 2 1 * *}$ & .20 \\
Bal3 & $8.6 \pm 3.6$ & $9.7 \pm 3.1$ & 0.33 & $\mathbf{. 0 0 6}$ \\
\hline
\end{tabular}

Note. $\quad d=$ Cohen's coefficient of effect size, $p=$ significance value of $t$-test. * medium practical significant difference, ${ }^{* *}$ large practical significant difference.

range of DCD prevalence (Kadesjö \& Gillberg, 1998; Lingam et al., 2009; Tsiotra et al., 2006; Wright \& Sugden, 1996). According to Zwicker, Missiuna, Harris, and Boyd (2012), the main reason for the high variability of DCD prevalence is different methods of identification. Higher prevalence often causes non-observance to all diagnostic criteria for specification of DCD in accordance with the norms (Geuze, Jongmans, Schoemaker, \& Smits-Engelsman, 2001; Zwicker et al., 2012). Sometimes in studies with children diagnosed as having DCD assessment of intelligence or the impact of motor difficulties on activities of daily life is missing (Geuze et al., 2001; Visser, 2003).

In comparison with previous studies focused on prevalence of DCD in children, the results of our study are encouragingly low. But despite a relatively low percentage of children with significant movement difficulty with high probability of presence of DCD, we found 26 children $(5.1 \%)$ who reached TTS $6-15^{\text {th }}$ percentile in the MABC-2 test, which denotes being at risk of having movement difficulties. Children at risk of having movement difficulties were also observed by Kourtessis et al. (2008) in 10.8\% of Greek children $(N=354)$, and by Schoemaker, Lingam, Jongmans, van Heuvelen, and Emond (2013) in 13.7\% of English children $(N=6959)$.

From the total of 507 children in our study, we found $33(6.5 \%)$ children with rDCD (22 boys and 11 girls; TTS $<15^{\text {th }}$ percentile). The odds ratio statistical method revealed almost twice as high predisposition $(O R=1.95,95 \%$ CI: 1.16-2.74) for the occurrence of movement difficulties in boys as compared to girls. In recent years, male female ratio with diagnosed DCD has decreased from 9:1 to 3:1 (Zwicker et al., 2012). However, current population studies state the male to female ratio with DCD is 2:1 or almost equal (Lingam et al., 2009). In children with movement difficulties with comorbid developmental or learning disorders, the gender differences are higher in boys, with a ratio of 2:1-3.4:1 (Pieters et al., 2012).

Nevertheless, in our study we have revealed relatively large gender differences in prevalence of significant movement difficulties with high probability of presence of DCD (six boys and one girl) with a relatively balanced number of boys and girls in the total sample of participants. Thus the results confirm that movement difficulties appear more often in boys than in girls, and with respect to the severity of these movement difficulties, the differences became higher. Our findings are consistent with those of Kourtessis et al. (2008), who observed a 5:1 male to female ratio with significant movement difficulties, and a 2:1 ratio in children at risk of having movement difficulties as indicated by the MABC test. Similarly, Lingam et al. (2009) detected differences in occurrence of being at risk of having movement difficulties (TTS $6-15^{\text {th }}$ percentile in MABC test) in boys and girls in the ratio $1.7: 1$, which is very close to our findings. However, in these authors' study, the male to female ratio with a total score below the $5^{\text {th }}$ percentile (which denotes significant movement difficulties) was much lower at 1.9:1 in comparison to our results.

In searching for gender differences in the individual motor coordination components in children with rDCD, we have revealed no statistically significant differences. However, we found girls to be better in manual 
dexterity (Cohen's $d=0.58$ ), while boys scored significantly better results in aiming and catching (Cohen's $d=0.50$ ). We have not observed any significant gender differences in balance skills.

Although the differences were not statistically significant, they reflect a general trend in gender differences concerning level of motor competence or level of motor skills. In order to get a better insight into the gender differences in motor skills, we studied the gender differences between the individual MABC-2 tests in the group of children with rDCD. In manual dexterity, girls achieved significantly better results in the MD1 test (drawing trail) as compared to boys (Cohen's $d=1.15 ; p \leq .05$; Table 4 ). In gross motor skills, in both tests (AC1 - catching with one hand, AC2 - throwing at wall target) boys achieved significantly better results (AC1 - Cohen's $d=0.98 ; p \leq .05$; AC2 - Cohen's $d=0.59)$. In balance, boys were significantly better in the Bal2 test (walking toe-to-heel backwards) (Cohen's $d=1.21 ; p \leq .05$ ). The statistically insignificant differences in manual dexterity and aiming and catching component of motor coordination could probably be caused by excessive difference in number of boys and girls in $\mathrm{rDCD}$ group. These results reflect the general trend in gender differences in motor skills. Girls are more likely to incline to activities that require fine motor skills as compared to boys. On the other hand, boys get more involved in activities that require manipulation with larger objects such as balls, which makes them better in gross motor activities (Booth et al., 2006; Junaid \& Fellowes, 2006). Apart from different levels of skills, these gender differences may be caused by a stronger social support and motivation to get involved in movement activities in favour of boys (Kourtessis et al., 2008). While creating the MABC test battery, Henderson and Sugden (1992) observed that girls were generally better in manual dexterity, while boys were better in ball skills. However, the results were not statistically significant. On the other hand, Kourtessis et al. (2008) noticed the same trend in their population study, with gender differences being statistically significant. Girls were significantly better in manual skills, while boys were significantly better in ball skills. The authors did not observe any significant differences in balance skills.

The results of this study suggest the current prevalence of developmentally conditioned movement difficulties with high probability of presence of DCD in older school-age Czech children. A probable limitation which may have a main effect on statistically insignificant confirmation of effect size in two components of motor coordination found in the present study may be the fact that many more boys than girls were included in the study.

\section{Conclusion}

Acquiring a sufficient level of motor skills in childhood is a basic precondition for participation in movement activities, optimal physical fitness and overall health and well-being. The results of our study have revealed significantly lower prevalence of significant movement difficulties with high probability of presence of DCD $(1.4 \%)$ in comparison with the prevalence quoted by the American Psychiatric Association (2-4\%) which is encouraging. Gender differences in children with rDCD in individual motor skills reflect a common trend in the general child population. Therefore, all intervention programmes created in order to support motor competence should respect these gender differences. Observance of all criteria for the utilization of the DCD diagnosis proves to be essential in detecting objective DCD prevalence.

\section{Acknowledgments}

The study has been supported by the Czech Science Foundation by projects No. P407/11/0946 and No. P39 Social-sciences aspects of human movement studies.

\section{References}

APA. (2000). Diagnostic and statistical manual of mental disorders (4th ed.). Washington, DC: Author.

APA. (2013). Diagnostic and statistical manual of mental disorders (5th ed.). Washington, DC: Author.

Barnett, L. M., Van Beurden, E., Morgan, P. J., Brooks, L. O., \& Beard, J. R. (2010). Gender differences in motor skill proficiency from childhood to adolescence: A longitudinal study. Research Quarterly for Exercise and Sport, 81, 162-170.

Barnhart, R. C., Davenport, M. J., Epps, S. B., \& Nordquist, V. M. (2003). Developmental coordination disorder. Physical Therapy, 83, 722-731.

Blank, R., Smits-Engelman, B., Polatajko, H., \& Wilson, P. (2012). European academy for childhood disability (EACD): Recommendations on the definition, diagnosis and intervention of developmental coordination disorder (long version). Developmental Medicine \& Child Neurology, 54, 54-93.

Booth, M., Okely, A. D., Denney-Wilson, E., Hardy, L., Yang, B., \& Dobbins, T. (2006). NSW schools physical activity and nutrition survey (SPANS) 2004 full report. Sydney, Australia: New South Wales Department of Health.

Butterfield, S. A., Angell, R. M., \& Mason, C. A. (2012). Age and sex differences in object control skills by children ages 5 to 14. Perceptual and Motor Skills, 114, 261-274.

Cermak, S. A., \& Larkin, D. (2002). Developmental coordination disorder. Albany, NY: Delmar. 
Chambers, M., \& Sugden, D. (2006). Early years movement skills: Description, diagnosis and intervention. London: Whurr Publisher.

Cheng, C. H., Ju, Y. Y., Chang, H. W., Chen, C. L., Pei, Y. C., Tseng, K. C., \& Cheng, H. Y. K. (2014). Motor impairments screened by the Movement Assessment Battery for Children-2 are related to the visual-perceptual deficits in children with developmental coordination disorder. Research in Developmental Disabilities, 35, 2172-2179.

Chow, S. M., Chan, L. L., Chan, C. P., \& Lau, C. H. (2002). Reliability of the experimental version of the Movement ABC. British Journal of Therapy and Rehabilitation, 9, 404-407.

Cohen, J. (1988). Statistical power analysis for the behaviour science (2nd ed.). New York, NY: Lawrence Erlbaum Associated.

Dorfberger, S., Adi-Japha, E., \& Karni, A. (2009). Sex differences in motor performance and motor learning in children and adolescents: An increasing male advantage in motor learning and consolidation phase gains. Behavioural Brain Research, 198, 165-171.

Dwyer, C., \& McKenzie, B. E. (1994). Impairment of visual memory in children who are clumsy. Adapted Physical Activity Quarterly, 11, 179-189.

Ehl, T., Roberton, M. A., \& Langendorfer, S. (2005). Does the throwing "gender gap" occur in Germany? Research Quarterly for Exercise and Sport, 76, 488-493.

Elbasan, B., Kayıhan, H., \& Duzgun, I. (2012). Sensory integration and activities of daily living in children with developmental coordination disorder. Italian Journal of Pediatrics, 38, 14.

Gaines, R., Missiuna, C., Egan, M., \& McLean, J. (2008). Interprofessional care in the management of a chronic childhood condition: Developmental coordination disorder. Journal of Interprofessional Care, 22, 552-565.

Geuze, R. H., Jongmans, M. J., Schoemaker, M. M., \& SmitsEngelsman, B. C. (2001). Clinical and research diagnostic criteria for developmental coordination disorder: A review and discussion. Human Movement Science, 20, 7-47.

Gillberg, C. (2003). Deficits in attention, motor control, and perception: A brief review. Archives of Disease in Childhood, 88, 904-910.

Goodway, J. D., \& Branta, C. F. (2003). Influence of a motor skill intervention on fundamental motor skill development of disadvantaged preschool children. Research Quarterly for Exercise and Sport, 74, 36-46.

Gur, R. C., Richard, J., Calkins, M. E., Chiavacci, R., Hansen, J. A., Bilker, W. B., ... Gur, R. E. (2012). Age group and sex differences in performance on a computerized neurocognitive battery in children age 8-21. Neuropsychology, 26, 251-265.

Haywood, K. M., \& Getchell, N. (2009). Life span motor development (5th ed.). Champaign, IL: Human Kinetics.

Henderson, S., \& Sugden, D. A. (1992). Movement Assessment Battery for Children. San Antonio, TX: Psychological Corporation.

Henderson, S. E., Sugden, D. A., \& Barnett, A. L. (2007). Movement Assessment Battery for Children-2. London: Harcourt Assessment.

Henniger, L. M. (2008). Teaching young children: An introduction (4th ed.). Upper Saddle River, NJ: Pearson.
Johnson, D. C., \& Wade, M. G. (2009). Children at risk for developmental coordination disorder: Judgement of changes in action capabilities. Developmental Medicine \& Child Neurology, 51, 397-403.

Junaid, K. A., \& Fellowes, S. (2006). Gender differences in the attainment of motor skills on the movement assessment battery for children. Physical and Occupational Therapy in Pediatrics, 26(1-2), 5-11.

Kadesjö, B., \& Gillberg, C. (1998). Attention deficits and clumsiness in Swedish 7-year-old children. Developmental Medicine \& Child Neurology, 40, 796-804.

Kadesjö, B., \& Gillberg, C. (1999). Developmental coordination disorder in Swedish 7-year-old children. Journal of the American Academy of Child \& Adolescent Psychiatry, 38, 820-828.

Kanioglou, A. (2006). Estimation of physical abilities of children with developmental coordination disorder. Studies in Physical Culture and Tourism, 13(2), 25-32.

Kourtessis, T., Tsougou, E., Maheridou, M., Tsigilis, N., Psalti, M., \& Kioumourtzoglou, E. (2008). Developmental coordination disorder in early childhood: A preliminary epidemiological study in Greek schools. International Journal of Medicine, 1, 95-99.

Lingam, R., Hunt, L., Golding, J., Jongmans, M., \& Emond, A. (2009). Prevalence of developmental coordination disorder using the DSM-IV at 7 years of age: A UK population-based study. Pediatrics, 123, 693-700.

Lorson, K. M., \& Goodway, J. D. (2008). Gender differences in throwing form of children ages 6-8 years during a throwing game. Research Quarterly for Exercise and Sport, 79, 174-182.

Macnab, J. J., Miller, L. T., \& Polatajko, H. J. (2001). The search for subtypes of DCD: Is cluster analysis the answer? Human Movement Science, 20, 49-72.

Malina, R. M., Bouchard, C., \& Bar-Or, O. (2004). Growth, maturation and physical activity. Champaign, IL: Human Kinetics.

Martin, N. C., Piek, J. P., \& Hay, D. (2006). DCD and ADHD: A genetic study of their shared aetiology. Human Movement Science, 25, 110-124.

Missiuna, C. (2001). Children with developmental coordination disorder: Strategies for success. Physical and Occupational Therapy in Pediatrics, 20(2-3), 1-4.

Missiuna, C., Gaines, R., McLean, J., Delaat, D., Egan, M., \& Soucie, H. (2008). Description of children identified by physicians as having developmental coordination disorder. Developmental Medicine \& Child Neurology, 50, 839-844.

Nolan, L., Grigorenko, A., \& Thorstensson, A. (2005). Balance control: Sex and age differences in 9 to 16 year olds. Developmental Medicine \& Child Neurology, 47, 449-454.

Pieters, S., de Block, K., Scheiris, J., Eyssen, M., Desoete, A., Deboutte, D., ... Roeyers, H. (2012). How common are motor problems in children with a developmental disorder: Rule or exception? Child: Care, Health and Development, 38, 139-145.

Pine, K. J., Lufkin, N., \& Messer, D. J. (2004). More gestures than answers: Children learning about balance. Developmental Psychology, 40, 1059-1067.

Poole, J. L., Burtner, P. A., Torres, T. A., McMullen, C. K., Markham, A., Marcum, M. L., ... Qualls, C. (2005). 
Measuring dexterity in children using the Nine-hole Peg Test. Journal of Hand Therapy, 18, 348-351.

Psotta, R., \& Hendl, J. (2012). Movement Assessment Battery for Children - second edition: Cross-cultural comparison between 11-15 year old children from the Czech Republic and the United Kingdom. Acta Universitatis Palackianae Olomucensis. Gymnica, 42(3), 7-16.

Psotta, R., Hendl, J., Frömel, K., \& Lehnert, M. (2012). The second version of the Movement Assessment Battery for Children: A comparative study in 7-10 year old children from the Czech Republic and the United Kingdom. Acta Gymnica, 42(4), 19-27.

Psotta, R., Kokštejn, J., \& Vodička, P. (2009). Nadváha a obezita u českých 11-14letých dětí s motorickými obtížemi a bez motorických obtíži [Overweight and obesity of Czech 11-14 years old children with and without motor difficulties]. Česká kinantropologie, 13(2), 75-83.

Schoemaker, M. M., Lingam, R., Jongmans, M. J., van Heuvelen, M. J., \& Emond, A. (2013). Is severity of motor coordination difficulties related to co-morbidity in children at risk for developmental coordination disorder? Research in Developmental Disabilities, 34, 3084-3091.

Schott, N., Alof, V., Hultsch, D., \& Meermann, D. (2007). Physical fitness in children with developmental coordination disorder. Research Quarterly for Exercise and Sport, 78, 438-450.

Thomas, J. R., \& French, K. (1985). Gender differences across age in motor performance. A meta-analysis. Psychological Bulletin, 98, 260-282.

Tsiotra, G. D., Flouris, A. D., Koutedakis, Y., Faught, B. E., Nevill, A. M., Lane, A. M., \& Skenteris, N. (2006). A comparison of developmental coordination disorder prevalence rates in Canadian and Greek children. Journal of Adolescent Health, 39, 125-127. van Beurden, E., Zask, A., Barnett, L. M., \& Dietrich, U. C. (2002). Fundamental movement skills: How do primary school children perform? The 'Move it Groove it' program in rural Australia. Journal of Science and Medicine in Sport, $5,244-252$.

Visser, J. (2003). Developmental coordination disorder: A review of research on subtypes and comorbidities. Human Movement Science, 22, 479-493.

Volman, J. M., \& Geuze, R. H. (1998). Relative phase stability of bimanual and visuomanual rhythmic coordination problems in children with a developmental coordination disorder. Human Movement Science, 17, 541-572.

Wann, J. P., Williams, M. M., \& Rushton, K. (1998). Postural control and co-ordination disorders: The swinging room revisited. Human Movement Science, 17, 491-513.

Watemberg, N., Waiserberg, N., Zuk, L., \& Lerman-Sagie, T. (2007). Developmental coordination disorder in children with attention-deficit-hyperactivity disorder and physical therapy intervention. Developmental Medicine \& Child Neurology, 49, 920-925.

Wright, H. C., \& Sugden, D. A. (1996). The nature of developmental coordination disorder: Inter and intragroup differences. Adapted Physical Activity Quarterly, 13, 357-371.

Wuang, Y. P., Su, J. H., \& Su, C. Y. (2012). Reliability and responsiveness of the Movement Assessment Battery for Children - second edition: Test in children with developmental coordination disorder. Developmental Medicine \& Child Neurology, 54, 160-165.

Zwicker, J. G., Missiuna, C., Harris, S. R., \& Boyd, L. A. (2012). Developmental coordination disorder: A review and update. European Journal of Paediatric Neurology, 16, 573-581. 